\title{
Poor Prognosis of Patients with Acute Myocardial Infarction Complicated by Diabetes Mellitus; Possible Role of Impaired Microvascular Function
}

Key words: myocardial infarction, diabetes mellitus, preinfarction angina, ischemic preconditioning

In January of 2001, The American Heart Association announced that the incidence and mortality rates for all forms of cardiovascular disease are 2- to 8-fold higher among individuals with diabetes than among those without diabetes, and that the in-hospital and long-term mortality rates following acute myocardial infarction (AMI) are twice as high among individuals with diabetes as among those without diabetes (1). Similar results were reported by the DARTS/MEMO collaboration in the United Kingdom (2), which found that the unadjusted two-year mortality rate ratio $(95 \% \mathrm{Cl})$ following a first $\mathrm{MI}$ in patients with type 2 diabetes and those without diabetes was $1.67(1.03-2.70)$. They also reported a significant trend toward increased mortality among younger patients with diabetes: Although there was no significant difference in death rates among those over 70 years of age, the death rate ratio was about 4.0 among diabetics under age 60 , and about 2.6 among diabetics aged $61-70$ years (2). In addition, a study from Spain showed 28-day mortality rates to be higher among MI patients with glycaemia $>6.67 \mathrm{mmol} / l$ on admission than among patients with lower levels (3). Notably, this increase in mortality was independent of all major confounding variables, including a previous diagnosis of diabetes.

Other mortality risk factors are also more prevalent among patients with diabetes. They are older; more likely to be female; more frequently have a history of MI, hypertension, hyperlipidemia and/or obesity; and are frequently complicated with renal dysfunction or peripheral or cerebrovascular disease. These patients may also suffer a greater degree of myocardial damage in AMI due, in part, to desensitization of pain receptors and prolongation of the interval from symptom onset to treatment (4). Impaired recognition of angina is particularly problematical in the setting of autonomic neuropathy in which sympathovagal imbalance lowers the threshold for life-threatening arrhythmias and increases the risk of hemodynamic instability (1). These patients also have higher heart rates and systolic blood pressures on admission (4), and have a greater frequency of 3-vessel disease and smaller reference diameter of the culprit lesion. Elevated fibrinogen and plasminogen activator inhibitor-1 levels and platelet abnormalities may increase the risk of thrombosis at the site of plaque disruption, thereby increasing the risk of reinfarction after reperfusion (1). Left ventricular remodeling is impaired and more likely to lead to congestive heart failure and cardiogenic shock. Finally, diabetic patients may be more likely to develop non-cardiac complications, including infections associated with invasive procedures and prolonged bed rest.

Still, the increased prevalence of comorbidities does not well explain the high mortality in AMI complicated by diabetes. Recently, however, the role of impaired microvascular function in these patients has become the focus of much attention. Iwakura et al (5) used intracoronary myocardial contrast echocardiography (MCE) to study the no-reflow phenomenon after successful reperfusion of AMI patients. They detected no reflow in $49(33.6 \%)$ of 146 patients studied. Because glucose levels in these patients were significantly higher on admission than in patients who did not exhibit this phenomenon $(209 \pm 79$ vs. $159 \pm 56 \mathrm{mg} / \mathrm{dl}$; $\mathrm{p}<$ 0.0001 ), it was concluded that hyperglycemia may be associated with impaired microvascular function following AMI. Furthermore, Kurisu et al (6) describe in this issue their measurement of the sum of the ST segment elevations ( $\Sigma S T)$ following successful myocardial reperfusion within 6 hours after onset in 134 patients with a first anterior AMI.

See also p 554.

Reduction of $\Sigma$ ST following reperfusion is a simple, reliable, noninvasive method for assessing the completeness of microvascular reperfusion (7). They found that the reduction in $\Sigma \mathrm{ST}$ after reperfusion was significantly smaller in diabetic patients, and that a complete lack of microvascular reperfusion occurred significantly more frequently among diabetics $(62.5 \%$ vs. $33.6 \%, p=0.01)$. They suggest several possible mechanisms to explain the association between diabetes mellitus and diminished microvascular reperfusion. Microvascular structural abnormalities, such as arteriolar thickening, perivascular accumulation of connective tissue and capillary microaneurysm, are often present in diabetic patients and could impair maximal coronary vasodilation. In addition, endothelium-dependent vascular relaxation is likely impaired in these patients, while ischemia/reperfusion- 
induced leukocyte accumulation and permeability increase and oxygen free radical production are likely all enhanced at the level of the microvascular circulation.

Because the no-reflow phenomenon is a benchmark of impaired microvascular function (8), and is a key determinant of functional and clinical outcomes following AMI, these reports may offer a better understanding of the diabetic heart, and represent an important milestone in the battle against heart disease.

Kinji IsHIKAWA, MD

Department of Cardiology, Kinki University School of Medicine, 377-2 Ohnohigashi, Osakasayama, Osaka 589-8511

\section{References}

1) Grundy SM, Howard B, Smith S Jr, Eckel R, Redberg R, Bonow RO. Prevention Conference VI: Diabetes and Cardiovascular Disease. Executive summary: Conference proceeding for healthcare professionals from a special writing group of the American Heart Association.
Circulation 105: 2231-2239, 2002.

2) Donnan PT, Boyle DIR, Broomhall J, et al. Prognosis following first acute myocardial infarction in type 2 diabetes: a comparative population study. Diabet Med 19: 448-455, 2002.

3) Sala J, Masiá R, González de Molina F-J, et al. Short-term mortality of myocardial infarction patients with diabetes or hyperglycaemia during admission. J Epidemiol Community Health 56: 707-712, 2002.

4) Angeja BG, de Lemos J, Murphy SA, et al. Impact of diabetes mellitus on epicardial and microvascular flow after fibrinolytic therapy. Am Heart J 144: 649-656, 2002.

5) Iwakura $\mathrm{K}$, Ito $\mathrm{H}$, Ikushima $\mathrm{M}$, et al. Association between hyperglycemia and the no-reflow phenomenon in patients with acute myocardial infarction. J Am Coll Cardiol 41: 1-7, 2003.

6) Kurisu S, Inoue I, Kawagoe T, et al. Diabetes mellitus is associated with insufficient microvascular reperfusion following revascularization for anterior acute myocardial infarction. Intern Med 42: 554-559, 2003.

7) Santoro GM, Valenti R, Buonamici P, et al. Relation between STsegment changes and myocardial perfusion evaluated by myocardial contrast echocardiography in patients with acute myocardial infarction treated with direct angioplasty. Am J Cardiol 82: 932-937, 1998.

8) Rezkalla SH, Kloner RA. No-reflow phenomenon. Circulation 105: 656-662, 2002. 